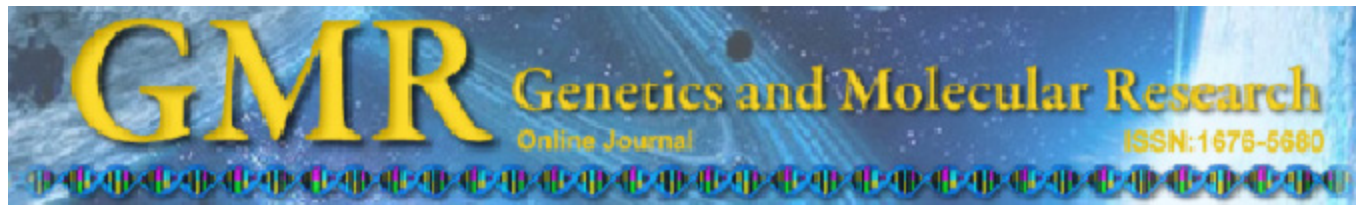

\title{
De novo DNA methylation of the paternal genome in 2-cell mouse embryos
}

\author{
X.S. Ma ${ }^{1}$, X.G. Wang ${ }^{1,2}$, L. Qin ${ }^{1}$, C.L. Song ${ }^{1}$, F. Lin ${ }^{1}$, J.M. Song ${ }^{1}$, \\ C.C. $\mathrm{Zhu}^{1}$ and H.L. Liu ${ }^{1}$ \\ ${ }^{1}$ College of Animal Science and Technology, Nanjing Agricultural University, \\ Nanjing, China \\ ${ }^{2}$ College of Animal Science, Xinjiang Agricultural University, Xinjiang, China \\ Corresponding author: H.L. Liu \\ E-mail: liuhonglin@njau.edu.cn
}

Genet. Mol. Res. 13 (4): 8632-8639 (2014)

Received March 25, 2013

Accepted December 6, 2013

Published October 27, 2014

DOI http://dx.doi.org/10.4238/2014.October.27.2

\begin{abstract}
The developmental dynamics of DNA methylation events have been well studied. Active demethylation of the paternal genome occurs in the zygote, passive demethylation occurs during cleavage stages, and de novo methylation occurs by the blastocyst stage. It is believed that the paternal genome has lower levels of methylation during early development than the maternal genome. However, in this study, we provide direct and indirect evidence of genome-wide de novo DNA methylation of the paternal genome after the first cell cycle in mouse embryos. Although very little methylation was detected within the male pronucleus in zygotes, an intense methylation signal was clearly visible within the androgenetic 2-cell embryos. Moreover, the DNA methylation level of the paternal genome in the post-zygotic metaphase embryos was similar to that of the maternal genome. Using indirect immunofluorescence with an antibody to methylated lysine 9 in histone $\mathrm{H} 3$, we provided new evidence to support the concept of spatial compartmentalization of parental genomes in 2-cell mouse embryos. Nevertheless, the transient segregation of parental genomes was not observed by determining the DNA methylation distribution in
\end{abstract}


the 2-cell embryos even though DNA methylation asymmetry between the maternal and paternal pronucleus existed in the 1-cell stage. The disappearance of separate immunofluorescence signals of 5-methyl cytosine in the 2-cell embryos might be attributed to the de novo methylation of the paternal genome during the first mitotic cycle.

Key words: DNA methylation; Paternal genome; Zygote; Mouse embryo

\section{INTRODUCTION}

DNA methylation at $\mathrm{CpG}$ dinucleotides is a major epigenetic modification of the genome, it plays an important role in regulating of gene expression, and it is essential for normal mammalian development (Robertson, 2005). Genomic methylation patterns in somatic differentiated cells are generally stable and heritable. However, genome-wide loss of DNA methylation is observed during early mouse development, reaching a low point during the blastocyst stage (Monk et al., 1987; Howlett and Reik, 1991; Kafri et al., 1992; Santos et al., 2002).

The earliest phase of this demethylation is confined to the paternal pronucleus prior to DNA replication (Mayer et al., 2000a; Oswald et al., 2000; Santos et al., 2002), and it has been described as "active" demethylation. The mechanism of active demethylation has been proposed recently, whereby 5 -methylcytosine $(5-\mathrm{MeC})$ is oxidized by ten-eleven translocation enzymes into 5-hydroxymethylcytosine (Guo et al., 2011; Ito et al., 2011). The maternal genome is demethylated by a passive mechanism that depends on DNA replication. The decreased DNA methylation in preimplantation embryos is thought to be because of the absence of the maintenance methylase, Dnmt1 (Howlett and Reik, 1991; Monk et al., 1991; Carlson et al., 1992; Rougier et al., 1998).

Although no direct evidence has been provided, it is believed that the paternal genome has lower methylation levels than the maternal genome during early development. However, in this study, we found that de novo DNA methylation occurred in androgenetic 2-cell embryos. The results suggested that de novo methylation of the paternal genome might happen during the passive demethylation of the maternal genome in early mouse development.

\section{MATERIAL AND METHODS}

All experiments were performed according to Nanjing Agricultural University Animal Care Committee guidelines, and all animal research was approved by the committee. All mice were killed by a standard protocol, and all efforts were made to minimize suffering.

\section{In vitro fertilization and embryo cultures}

Female ddY mice, 21-23 days old, were superovulated with 5 IU pregnant mare's serum gonadotropin, which was followed $48 \mathrm{~h}$ later with $5 \mathrm{IU}$ human chorionic gonadotropin (hCG). Unfertilized metaphase II-arrested oocytes were collected in Whitten's medium from the ampullae of oviducts 14-15 $\mathrm{h}$ after hCG injection. Sperm were collected from the caudal epididymis of adult ICR males and were preincubated in Whitten's medium for $2 \mathrm{~h}$ in an atmosphere of $5 \% \mathrm{CO}_{2}, 95 \%$ air at $38^{\circ} \mathrm{C}$. The oocytes were inseminated with capacitated sperm. 
Three hours after insemination, the fertilized oocytes were washed and cultured in CZB medium (Chatot et al., 1990).

\section{Parthenogenesis and androgenesis}

Androgenetic embryos were prepared by fertilizing enucleated oocytes. Cumulusoocyte complexes were collected from the ampullae of oviducts $14-15 \mathrm{~h}$ after hCG injection and were placed in KSOM (Lawitts and Biggers, 1993) that contained $0.3 \mathrm{mg} / \mathrm{mL}$ bovine testicular hyaluronidase. After complete removal of the cumulus cells, the oocytes were transferred to a micromanipulation drop, which contained HEPES-buffered KSOM that was supplemented with $5 \mu \mathrm{g} / \mathrm{mL}$ cytochalasin B. The zona pellucida was permeabilized by a Piezo-impact-driven micromanipulator (Prime Tech Ltd, Ibaraki, Japan), and the metaphase II chromosomes were aspirated with a minimal volume of oocyte cytoplasm using a pipette. The enucleated oocytes were transferred to acidic MEMCO for a short time to dissolve the zona pellucida. Zona-free oocytes were cultured in Whitten's medium for $1 \mathrm{~h}$ to allow them to recover after zona pellucida removal, and they were then subjected to in vitro fertilization. Thirty minutes after insemination, the oocytes were washed and cultured in CZB medium. Parthenogenetically activated oocytes were prepared by exposing oocytes at the metaphase II stage to 7\% ethanol in CZB for 6 min. In order to obtain diploid parthenogenetic embryos, the oocytes were cultured in CZB that contained $5 \mu \mathrm{g} / \mathrm{mL}$ cytochalasin B for $6 \mathrm{~h}$ and then were cultured in CZB.

\section{Production of post-zygotic metaphase (PZM) embryos}

A new protocol was used to produce the embryos that occurred after inhibiting the parental genomes from mingling and cytokinesis during the first cell cycle to form a single cell that contained male and female nuclei in a common cytoplasm (we called these embryos PZM). Embryos with 2 pronuclei $10 \mathrm{~h}$ after insemination were selected and cultured in CZB medium containing $0.5 \mu \mathrm{g} / \mathrm{mL}$ nocodazole to arrest the cell cycle at $\mathrm{M}$ phase. Four hours later, $8 \mathrm{mM}$ 6-dimethylaminopurine (6-DMAP) was added to the medium. Nuclei formed after being cultured for $3 \mathrm{~h}$ in the presence of nocodazole and 6-DMAP. Then, the embryos were washed and cultured in CZB for $5 \mathrm{~h}$ before being subjected to immunocytochemistry.

\section{Immunocytochemistry}

Oocytes and embryos were washed in phosphate-buffered saline (PBS) that contained $3 \mathrm{mg} / \mathrm{mL}$ polyvinylpyrrolidone, fixed for $1 \mathrm{~h}$ in $3.7 \%$ paraformaldehyde in PBS, and permeabilized with $0.5 \%$ Triton X-100 in PBS for $20 \mathrm{~min}$ at room temperature. The cells were incubated for $1 \mathrm{~h}$ with a 1:200 dilution of the rabbit polyclonal antibody that recognizes dimethyl-lysine 9 on histone H3 (Upstate Biotechnology, Lake Placid, NY, USA) and a secondary fluorescein isothiocyanate (FITC)-conjugated antibody (Jackson Immunoresearch, West Grove, PA, USA). To detect 5-MeC, the cells were treated with $2 \mathrm{~N} \mathrm{HCl}$ at room temperature for $30 \mathrm{~min}$ and were

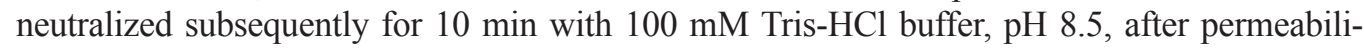
zation. After extensive washing with $0.05 \%$ Tween-20 in PBS, the cells were incubated with anti-5-MeC antibodies (Eurogentec, Seraine, Belgium), followed by incubation with secondary FITC-conjugated antibody. DNA was stained with $3 \mu \mathrm{g} / \mathrm{mL}$ 4,6-diamidino-2-phenylindole 
(DAPI) for $20 \mathrm{~min}$, and the cells were mounted on a glass slide in Vectashield anti-bleaching solution (Vector Laboratories, Burlingame, CA, USA). Fluorescence was detected using a Zeiss LSM700 laser-scanning confocal microscope.

\section{RESULTS}

\section{Demethylation and de novo methylation of the paternal genome in early mouse embryos}

Genomic methylation was visualized using indirect immunofluorescence with an antibody to $5-\mathrm{MeC}$. The reliability of this technique has been firmly established by a number of independent studies (Reynaud et al., 1992; Tweedie et al., 1997; Santos et al., 2002). Previous studies showed the asymmetric loss of methylation by the male pronucleus after fertilization. The loss of paternal methylation begins very quickly after the removal of protamine, and the process of active demethylation takes place very rapidly, resulting in the almost complete loss of methylation from the male pronucleus. The maternal DNA is more highly methylated than the paternal DNA in zygotes moving through syngamy; however, the paternal genome does have a low but significant positive methylation signal at syngamy (Santos et al., 2002). Does de novo methylation happen in the paternal genome during the first cell cycle? In an attempt to understand this process, we investigated the DNA methylation reprogramming in androgenetic and parthenogenetic embryos. In order to strictly compare and evaluate the methylation status of individual embryos during this phase, it was important that all stages were treated and stained together on the same slide. These results show that very little methylation was detected within the male pronucleus in zygotes; however, an intense signal was clearly visible within the androgenetic 2-cell embryos, demonstrating that de novo methylation of paternal genomes takes place after the first mitotic division (Figure 1). In addition, no de novo DNA methylation was observed in the paternal genome in the delayed 1-cell embryos that were treated with aphidicolin within 6-24 h of insemination (the embryos corresponded to G2 of the 2-cell stage). This suggested that the de novo DNA methylation of the paternal genome depended on the first cell cycle. Through observation of the methylation pattern of embryos, we also found that patterns of methylation staining throughout the nucleus were even, but an uneven distribution was observed in androgenetic 4-cell embryos.

\section{Disappearance of asymmetric DNA methylation patterns between paternal and maternal genomes in PZM embryos}

In order to confirm the result that de novo DNA methylation happened in the paternal genome after the first cell cycle, we examined the DNA methylation pattern in PZM embryos. As we described previously, PZM embryos occurred after inhibiting the parental genomes from mingling and cytokinesis during the first cell cycle to form a single cell that contained male and female nuclei in a common cytoplasm (Liu et al., 2005). The 2 nuclei in PZM embryos were derived from the male and female genomes because the asymmetry of histone H3 lysine 9 (H3K9) methylation between the zygotic male and female pronuclei remained in the 2 nuclei. The DNA methylation fluorescence between paternal and maternal genomes in the PZM embryos was similar (Figure 2), and this supported the finding that de novo DNA meth- 
ylation had taken placed in the paternal genome after the first cell cycle. Moreover, the de novo DNA methylation of the paternal genome did not depend on the cytokinesis of the first mitosis.

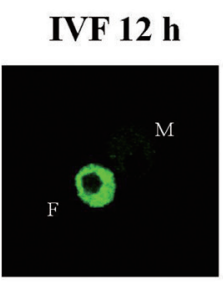

G-2C

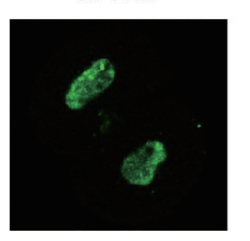

Aphi 6-24 h

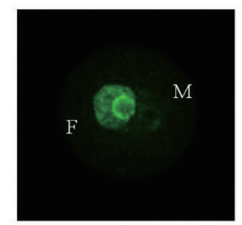

A-4C

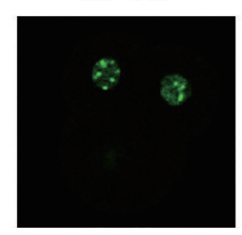

A-2C

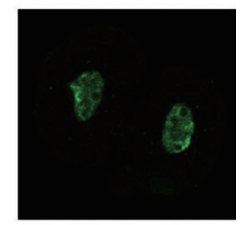

G-4C

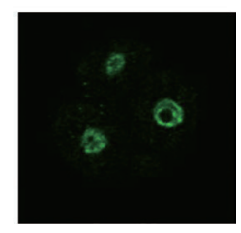

Figure 1. DNA methylation patterns in fertilized, androgenetic and parthenogenetic mouse embryos. Mouse fertilized 1-cell embryos, parthenogenetic and androgenetic 2- and 4-cell embryos were subjected to immunocytochemistry with the antibody to DNA cytosine methylation $(5-\mathrm{MeC})$. The antibody was localized with FITC-conjugated secondary antibodies (green). Fertilized 1-cell embryos were collected $12 \mathrm{~h}$ after in vitro fertilization (IVF $12 \mathrm{~h}$ ) or collected $24 \mathrm{~h}$ after an incubation with Aphicicolin in a period of 6-24 h after in vitro fertilization (Aphi 6-24 h). A-2C and A-4C indicate androgenetic 2- and 4-cell embryos, respectively; G-2C and G-4C indicate parthenogenetic 2and 4-cell embryos, respectively. $\mathrm{M}$ represents male pronucleus, $\mathrm{F}$ represents female pronucleus. The experiments were conducted at least two times and similar results were obtained.

\section{6-DMAP}

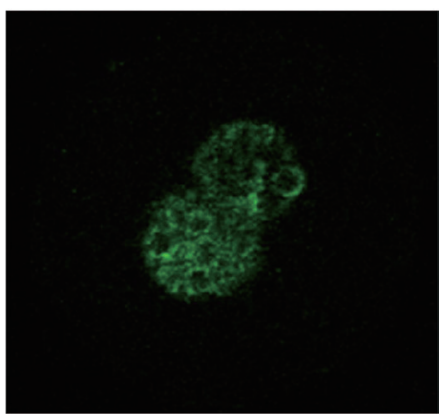

Figure 2. DNA methylation fluorescence in PZM mouse embryos. PZM embryos were wash and cultured in CZB for $5 \mathrm{~h}$. The PZM embryos were subjected to immunocytochemistry with the antibody to DNA cytosine methylation $(5-\mathrm{MeC})$. The antibody was localized with FITC-conjugated secondary antibodies (green). The experiments were conducted three times and similar results were obtained.

\section{Indirect evidence showing de novo DNA methylation of the paternal genome in 2-cell embryos}

After fertilization, the paternal genome is demethylated by an active mechanism prior 
to DNA replication, and it results in asymmetric DNA methylation between the paternal and maternal genomes (Mayer et al., 2000a; Oswald et al., 2000; Santos et al., 2002). Using indirect immunofluorescence with an antibody to $5-\mathrm{MeC}$, the topological separation of paternal and maternal genomes in early mouse embryos has been suggested (Mayer et al., 2000a,b). A transient segregation of parental genomes is preserved up to the 4-cell embryo stage, and then it gradually disappeared. However, the concept of spatial separation of parental genomes is not supported by a more recent report that used the same antibody (Santos et al., 2002). To argue the discrepancy, the succeeding researchers point out that the spatial separation of the parental genomes might result from a harsh fixation protocol. In the present study, the immunofluorescence of 5-MeC appeared evenly in the nuclei of 2-cell embryos without any evidence of spatial compartmentalization. However, we did observe spatial separation of the parental genomes in 2-cell mouse embryos using indirect immunofluorescence with an antibody to methylated H3K9. We used antibody that specifically recognized dimethylated $\mathrm{H} 3 \mathrm{~K} 9$ to examine the $\mathrm{H} 3 \mathrm{~K} 9$ methylation state in mouse early embryos. The male pronucleus was not stained or showed very weak fluorescence for $\mathrm{H} 3 \mathrm{~K} 9$ methylation, and the female pronucleus showed a clear methylation signal (Figure 3). The asymmetric H3K9 methylation between the paternal genome and maternal genome persisted throughout the 1-cell stage of embryos. These observations were confirmed using parthenogenetic and androgenetic embryos (Liu et al., 2004). Intense methylation fluorescence only appeared in parthenogenetic 2-cell embryos, and methylation fluorescence was not present or was very weak in androgenetic 2-cell embryos. In the normal 2-cell embryos, spatial separation of the H3K9 methylation distribution was observed in the present study; we also observed the spatial compartmentalization of the paternal and maternal genomes at anaphase of the first mitosis (Figure 3). Our results supported the concept of spatial compartmentalization of parental genomes in 2-cell embryos; the disappearance of separate 5-MeC immunofluorescence signals in the 2-cell embryos might be attributed to the de novo methylation of the paternal genome during the first mitosis.

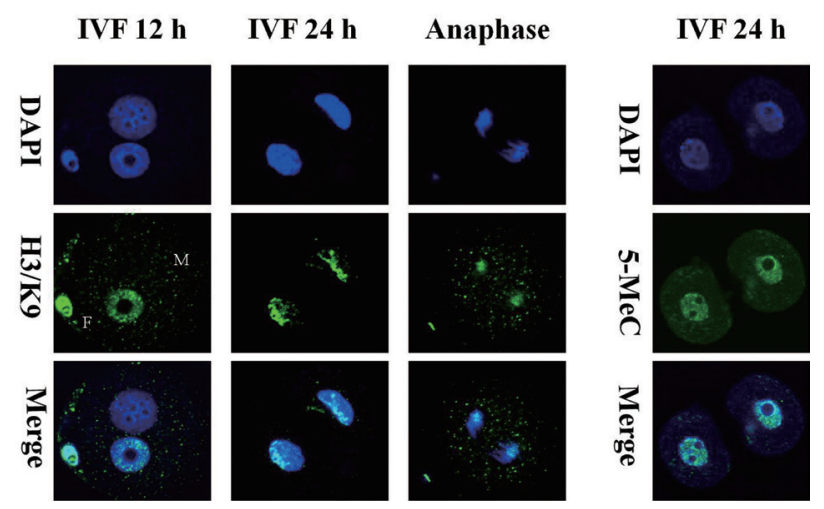

Figure 3. De novo DNA methylation of paternal genome is confirmed by the distribution of parental genomes in 2-cell mouse embryos. Topological separation of paternal and maternal genomes in 2-cell mouse embryos has been observed by using indirect immunofluorescence with an antibody to methylated lysine 9 in histone H3. However, spatial separation of parental genomes becomes undetectable using antibody to DNA cytosine methylation (5$\mathrm{MeC}$ ). The disappearance of the separation might result from de novo DNA methylation of paternal genomes. The embryos were collected at 12, 17, and $24 \mathrm{~h}$ after insemination, designated as samples IVF $12 \mathrm{~h}$, Anaphase, and IVF $24 \mathrm{~h}$, respectively, and subjected to immunocytochemistry using the antibody against methylated histone H3 lysine 9, or the antibody against DNA cytosine methylation (5-MeC). The antibodies were localized with FITC-conjugated secondary antibodies (green), and the DNA was stained with DAPI (blue). The experiments were conducted at least two times and similar results were obtained. 


\section{DISCUSSION}

The developmental dynamics of DNA methylation events have been well studied. Active demethylation of the paternal genome occurs in the zygote and is followed by passive demethylation during cleavage stages; de novo methylation occurs by the blastocyst stage. Although the active demethylation event results in the almost complete loss of methylation from the male pronucleus, there is a small but significant residual methylation signal in some but not all of the paternally derived chromosomes at syngamy (Santos et al., 2002). Studies have also shown that parent-specific methylation imprinting of some genes is established during or after fertilization (El-Maarri et al., 2001; Pickard et al., 2001; Kim et al., 2004; Wossidlo et al., 2010). These observations imply that de novo DNA methylation events of the paternal genome might happen after the first mitosis. In this study, we showed that genome-wide de novo DNA methylation of the paternal genome occurred after the first cell cycle. The de novo methylation reprogramming may have roles in imprinting, controlling gene expression, and establishing nuclear totipotency.

Several lines of evidence provided by Mayer et al. $(2000 \mathrm{a}, \mathrm{b}, \mathrm{c})$ suggest the existence of separate nuclear compartments for the paternal and maternal genomes during preimplantation development, when chromatin remodeling and epigenetic reprogramming occur. However, a discrepant result was reported by Santos et al. (2002). Santos et al. argue that the different conclusions may be because of the preparative techniques, which employed a harsh fixation protocol that results in significant embryo loss. New evidence is required to confirm the concept of spatial separation of the parental genomes in mouse early embryos. Using immunofluorescence with antibody to methylated $\mathrm{H} 3 \mathrm{~K} 9$, we observed the spatial compartmentalization of the parental genomes. The role of the protocol to detect the H3K9 methylation state should be kept in mind because no special treatment was adopted in it. Separate nuclear compartments might be functionally important, involved in epigenetic reprogramming, and have important implications for the establishment of genetic totipotency in the early embryo. It may explain why attempts to clone animals often fail. The lack of asymmetry in a transplanted somatic cell nucleus might confuse the reprogramming machinery of the oocytes.

\section{ACKNOWLEDGMENTS}

Research supported by the Key Project of Chinese National Natural Science Fund (\#31072028) and the Chinese National Programs for Fundamental Research and Development (“973" Program \#2007CB947403). We would like to thank Professor Fugaku Aoki of Tokyo University, Japan, for the mouse embryo culture protocols.

\section{REFERENCES}

Carlson LL, Page AW and Bestor TH (1992). Properties and localization of DNA methyltransferase in preimplantation mouse embryos: implications for genomic imprinting. Genes Dev. 6: 2536-2541.

Chatot CL, Lewis JL, Torres I and Ziomek CA (1990). Development of 1-cell embryos from different strains of mice in CZB medium. Biol. Reprod. 42: 432-440.

El-Maarri O, Buiting K, Peery EG, Kroisel PM, et al. (2001). Maternal methylation imprints on human chromosome 15 are established during or after fertilization. Nat. Genet. 27: 341-344.

Guo JU, Su Y, Zhong C, Ming GL, et al. (2011). Emerging roles of TET proteins and 5-hydroxymethylcytosines in active DNA demethylation and beyond. Cell Cycle 10: 2662-2668. 
Howlett SK and Reik W (1991). Methylation levels of maternal and paternal genomes during preimplantation development. Development 113: 119-127.

Ito S, Shen L, Dai Q, Wu SC, et al. (2011). Tet proteins can convert 5-methylcytosine to 5-formylcytosine and 5-carboxylcytosine. Science 333: 1300-1303.

Kafri T, Ariel M, Brandeis M, Shemer R, et al. (1992). Developmental pattern of gene-specific DNA methylation in the mouse embryo and germ line. Genes Dev. 6: 705-714.

Kim SH, Kang YK, Koo DB, Kang MJ, et al. (2004). Differential DNA methylation reprogramming of various repetitive sequences in mouse preimplantation embryos. Biochem. Biophys. Res. Commun. 324: 58-63.

Lawitts JA and Biggers JD (1993). Culture of preimplantation embryos. Meth. Enzymol. 225: 153-164.

Liu H, Kim JM and Aoki F (2004). Regulation of histone H3 lysine 9 methylation in oocytes and early pre-implantation embryos. Development 131: 2269-2280.

Liu HL, Hara KT and Aoki F (2005). Role of the first mitosis in the remodeling of the parental genomes in mouse embryos. Cell Res. 15: 127-132.

Mayer W, Niveleau A, Walter J, Fundele R, et al. (2000a). Demethylation of the zygotic paternal genome. Nature 403: 501-502.

Mayer W, Smith A, Fundele R and Haaf T (2000b). Spatial separation of parental genomes in preimplantation mouse embryos. J. Cell Biol. 148: 629-634.

Mayer W, Fundele R and Haaf T (2000c). Spatial separation of parental genomes during mouse interspecific (Mus musculus x M. spretus) spermiogenesis. Chromosome. Res. 8: 555-558.

Monk M, Boubelik M and Lehnert S (1987). Temporal and regional changes in DNA methylation in the embryonic, extraembryonic and germ cell lineages during mouse embryo development. Development 99: 371-382.

Monk M, Adams RL and Rinaldi A (1991). Decrease in DNA methylase activity during preimplantation development in the mouse. Development 112: 189-192.

Oswald J, Engemann S, Lane N, Mayer W, et al. (2000). Active demethylation of the paternal genome in the mouse zygote. Curr. Biol. 10: 475-478.

Pickard B, Dean W, Engemann S, Bergmann K, et al. (2001). Epigenetic targeting in the mouse zygote marks DNA for later methylation: a mechanism for maternal effects in development. Mech. Dev. 103: 35-47.

Reynaud C, Bruno C, Boullanger P, Grange J, et al. (1992). Monitoring of urinary excretion of modified nucleosides in cancer patients using a set of six monoclonal antibodies. Cancer Lett. 61: 255-262.

Robertson KD (2005). DNA methylation and human disease. Nat. Rev. Genet. 6: 597-610.

Rougier N, Bourc'his D, Gomes DM, Niveleau A, et al. (1998). Chromosome methylation patterns during mammalian preimplantation development. Genes Dev. 12: 2108-2113.

Santos F, Hendrich B, Reik W and Dean W (2002). Dynamic reprogramming of DNA methylation in the early mouse embryo. Dev. Biol. 241: 172-182.

Tweedie S, Charlton J, Clark V and Bird A (1997). Methylation of genomes and genes at the invertebrate-vertebrate boundary. Mol. Cell. Biol. 17: 1469-1475.

Wossidlo M, Arand J, Sebastiano V, Lepikhov K, et al. (2010). Dynamic link of DNA demethylation, DNA strand breaks and repair in mouse zygotes. EMBO J. 29: 1877-1888. 